\title{
The Application of Digital Technology in the Protection and Inheritance of the Intangible Cultural Heritage of the Martial Arts
}

\author{
Yin Tenglong \\ Public P. E. Department of Luoyang Normal University, Henan, Luoyang, 471022, P.R.China
}

13403792438@163.com

\begin{abstract}
Keywords: martial arts; intangible cultural heritage; digital; modular forms
Abstract: at present, the inheritance of the intangible cultural heritage of the martial arts has been in a fix, but the application of digital technology provides a new way for the development of the martial arts. The digital technology system of the intangible cultural heritage is a digital service platform which includes digital consumption and the collection, storage, management, production and communication of digital resources. Each link is supported by the corresponding technique, which means all the links are existed as independently modular forms and can be promoted in proper sequence. The digital methods has expanded the space of propagation, enriched the ways of transmission, and enhanced the efficiency and effect for the protection and inheritance of the intangible cultural heritage of the martial arts.
\end{abstract}

\section{Research Methods}

\subsection{Information Research Method}

Using the principle of information theory, system theory and control theory. The information of martial arts as intangible cultural inheritage has been collected, classified, stored, processed, spread, and finally applied in practice, in order to realize the dynamic protection of traditional martial arts.

\subsection{Simulation Method}

Using digital module technology and combining with the characteristics of the martial arts, to build a digital protection and heritage system for the martial arts as intangible cultural heritage.

\section{The Advantages and Characteristics of the Digital Protection of the Intangible Cultural Heritage of the Martial Arts}

\subsection{High Speed}

The Internet, as the carrier of the digital communication method, eliminates the procedures for the traditional media like printing and publishing, distribution and sale of links. With the improvement of the software and hardware technology of the internet, the propagation delay of the information of martial arts in time and space can even be ignored, which truly achieves global synchronization.

\subsection{Large Capacity}

The kinds of information sources of martial arts are more extensive, the means of transmission is more abundant, and the content of information is more vast and diversified. Due to the restriction of the carrier and the spreading media, the capacity of the traditional media is limited, while the digital propagation has benefited from the rapid development of information technology, which has approved to infinite capacity and propagative ability.

\subsection{Interactiveness}

Digital technology has broken the limitation of traditional forms of information dissemination of martial arts. Mass media has unified the 3 different ways of spreading, peer-to-peer, groups-to-groups and peer-to-groups. The Internet is both a tool which is used for mass communication to convey information to the audience to teach them martial arts and a way that is provided for interaction and communication among the enthusiasts. 


\subsection{Opening and Compatibility}

The martial arts in the digital era has blurred the concept of time and space, which bringing about the full range of different regions, countries and social cultures. Regardless of cultural background, performance form and display contents, all the information of the martial arts can get the platforms and opportunities to display and exchange, which means various forms of martial arts can coexist.

\section{The Way of Protecting the Intangible Cultural Heritage of the Martial Arts}

The digital protection of the Martial Arts is the transformation of Martial Arts itself. "The complicated and messy information of martial arts can be transformed into some measureable figures and data. Then a digital model will be established basing on them, which can measure the information uniformly with both keeping the essence and adapting the social development. ${ }^{[1]}$ It has been an inevitable choice to apply the digital technology to protecting and developing the intangible cultural heritage of the martial arts in digital era. Through the digital platform, people can use a variety of channels to understand the traditional martial arts and related knowledge, which can also display a number of traditional martial arts and boxing, that have long history, maybe even on the verge of extinction, in front of more people and expand the audience of traditional martial arts. The digital way has expanded the propagation space of martial arts, and has attracted more and more fans to participate in the protection and inherit of the traditional martial arts.

\section{The Protection Technology and Products of the Intangible Cultural Heritage of the Martial Arts}

The digital products of the intangible cultural heritage of martial arts mainly refers to those which are developed by using abundant texts, images, videos and three-dimensional models in the resource database and with the theme of the intangible cultural heritage of martial arts. With the development and popularization of multi-touch screen, tablet PC, multimedia interactive TV and other receiving terminals, the form of digital products gets into a new media era. The new media becomes an important carrier and way of updating the technology of digital cultural industry and renewing the means of transmission gradually. The biggest difference between new media and traditional media is the enhancement of interactivity which motivates and interests the audience apprantly. Therefore, in the field of digital products development, the new media digital products that applied with virtual reality technology, human-computer interaction technology, three-dimensional display technology and other emerging information technologys are favored. It has already been the trend of development and mainstream to comprehensively and gradually apply various forms by using the digital products like three-dimensional, interactive and other new kind of media in displaying the connotation of the intangible cultural heritage of martial arts.

\section{The Construction of Digital Protection Technology System of the Intangible Cultural Heritage of Martials Arts}

"The digital protection technology system of the intangible cultural heritage of martial arts includes five modules, from the digital collection, digital storage, digital management, digital production to digital consumption". ${ }^{2]}$ Each part is supported by the corresponding technique, which are mutually independent and can be carried out sequently. At last, the digital consumption will expand the living space, achieve the actively dynamic protection of the intangible cultural heritage of the martial arts, and creat the material basis and social environment for the ecological cycle of digital protection.

\subsection{Digital Acquisition Module of the Intangible Cultural Heritage of Martial Arts}

To excavate the connotation of the intangible cultural heritage of the martial arts by using a variety of techniques and norms for digital collection. Digital acquisition technology is mainly 
refers to the digital record, processing and reappearance that are applied with a certain collection technology in the intangible cultural heritage of the martial arts, and the related environment, space and artifacts.

Compared with the traditional video recording, audio capture and text scanning, the ways of digital collection is not only a simple record, but also including the application of a variety of techniques, such as 3D motion capture, 3D modeling, environmental simulation technology and other techniques to recreat the the digital technology of the reasources of the intangible cultural heritage of martial arts.All of the exploration before is aimed at recording the living and develpoment enviroment of the traditional martial arts, enriching the traditional single video recording method, realizing the stereoscopic inheritance of the intangible cultural inheritage, breaking through and creating the forms and meathods of the digital performance.

\subsection{Digital Memory Module of the Intangible Cultural Heritage of the Martial Arts}

Accroding to the characteristics, like rich contents, mutiple representation forms, and various types of categories, of the intangible cultural inheritage of the martial arts, appropriate classification techniques have to be applied in the process of effective digital storage, which can also reduce the occurrence of storage duplication and omission. Therefore, it must be solved firstly about the problems of the standard of digital resources representation and the digital classification, which is the premise of systematizing the digital storage. The standard of digital representation is to unify the technical standards in the process of the resources Digital processing. There should be a resources technical standard that is in accordance with the different places in China, and that can even get the common recognition around the Chinese culture circle. And the standard should contain the technical standards of resource construction, resource description, resource organization, resource management and resource preservation. Digital classification is mainly based on the characteristics of gradualness and systematicness of the intangible cultural heritage. It has studied the composition and classification of intangible cultural heritage of martial arts, from the interdisciplinary angle of sociology, history, and cultural heritage. And the digital classification system is set accroding to the wide geographical distribution and a great deal of information of intangible cultural inheritage.

\subsection{The Digital Production Module of the Intangible Cultural Heritage of Martial Arts}

The resources need to be producted as digital products with the comprehensive application of computer graphics, image processing, computer animation, and the establishment of a complex dye scene. The traditional protection of intangible cultural heritage of martial arts is mostly based on the static record and storage, which actually saving more than developing.It can not solve the problem of the development of intangible cultural heritage of martial arts fundamentally. The digital production technology distributes the resource again,making the consumer's demand as the guide. It transfers intangible cultural heritage of the martial arts into the available material, through the creation, production and marketing, casting it to the market in the form of products , with economic benefits to promote heritage protection and inheritance. Digital martial arts heritage includes film, animation, documentary and games, which displays with the interaction of virtual-reality and human-computer. It attracts different groups, different ages of the audience to participate with the various forms of products. It guides people from cognition, attention to conscious protection and inheritance, and develops intangible cultural heritage of martial arts the dynamicly and actively.

\subsection{Digital Consumption Module of the Intangible Cultural Heritage of Martial Arts}

Through computer, mobile phone, digital TV and other terminals, in the form of multimedia display, human-computer interaction in the digital theater, digital tourism and other areas of digital consumption, Digital consumption module help to obtain a certain economic benefits. Both of the digital communication of the intangible cultural heritage of martial arts and the consumer technology system which including satellite TV, IPTV and multi terminal display technology and other communication technology, are spread by digital display entities in digital museum. The digital consumer technology system mainly involves the control technology, the sound photoelectric 
technology, the multimedia display technology, and so on. As in theater stage through digital fusion of sound photoelectric technology, digital products and real stage could be perfect, which can make the audience in the augmented reality scene in a virtual environment and to feel personally on the scene, especially in Shaolin Temple "Zen Shaolin• Music Ceremony".

\section{Conclusion}

The digital protection and development technology system need to be constructed by using the modern techniques and computer technology to collect, store, manage, product, spread and consume the resources of the intangible cultural heritage of martial arts digitally. The connotation and enchantment of the martial arts can be propagated by building digital martial information resource base and developing relevant digital products, to show the public the intangible cultural heritage of martial arts in plenty of ways. ${ }^{[3]}$ In the initial stage, there are some unsatisfactory aspects, such as digital acquisition and product form, however, the digital martial arts has demonstrated a bright future. This is not only an important content of sports cultural information construction, but also an important way to enhance the soft power of the national culture, what is more, this is an important measure to protect the cultural diversity of the Chinese nation.

\section{Acknowledgements}

Project: 2016 Soft Science Project of Science and Technology Department in Henan Province; Project name: A Research on the Development of Networking Technology and Market Application for "Living” Heritage of Central Plains Martial Arts as Intangible Cultural Heritage.

\section{References}

[1] Huang Yonglin, Tan Guoxin. Research on Digital Protection and Development of Intangible Cultural Heritage in China [J]. Central China Normal University Academic Journal (Humanities and Social Sciences), 2013, 51 (2): 49

[2] Sun Chuanming. Research on Digital Technology of Intangible Cultural Heritage of Ethnic Dance [D].Wuhan, Central China Normal University, 2013:25-28

[3] Yu Dinghai, Niu Aijun. Research on the Inheritance of Chinese Martial Arts [M]. Beijing, Physical Education Press, 2010:91-92 